\title{
Basedow-Graves' disease in a pediatric patient with Sticlker syndrome, a new endocrine finding to improve personalized treatment
}

\author{
Roberta Onesimo ${ }^{1,2}$, Cristina De Rose ${ }^{2^{*}}$, Clelia Cipolla ${ }^{2}$, Silvia Della Casa ${ }^{3,4}$, Chiara Leoni ${ }^{1,2}$, Annabella Salerni ${ }^{4,5}$,
} Daniela Ricci ${ }^{6,7}$ and Giuseppe Zampino ${ }^{1,4}$

\begin{abstract}
Background: Stickler syndrome is a connective tissue disorder with predominantly autosomal dominant inheritance, with ocular, auditory and joint involvement.

Thyroid dysfunction was not described as part of alterations in Stickler syndrome and in particular, the association between Stickler's syndrome and Graves' disease has never been previously reported in literature.

Moreover, the presence of Graves' disease is uncommon in the pediatric age (especially in children younger than 6 years old).

Case presentation: We report the case of a 5-years old child affected by Stickler syndrome who received the diagnosis of Graves's disease, in absence of suggestive symptoms, during health supervision.

Conclusions: This is the first evidence of thyroid dysfunction and autoimmune pattern for Sticker syndrome. Further clinical reports are expected before suggesting the implementation of new clinical skills for Stickler syndrome, but this paper may contribute to improve personalized management of this rare disorder.
\end{abstract}

Keywords: Stickler syndrome, Basedow-Graves's disease, Pediatric disability, Autoimmune disease, Nutritional assessment

\section{Introduction}

Stickler syndrome (SS) is a rare genetically heterogeneous disorder of the connective tissue, caused by abnormal synthesis of type II, XI, or IX collagen [1]. We distinguish different syndrome subtypes: type I, the most common, due to mutations in the COL2A1 gene; type II, due to mutation of the COL11A1 gene; type III caused by mutations in the COL11A2 gene. These three subtypes exhibit an autosomal dominant mode [1].

\footnotetext{
* Correspondence: cristyderose@gmail.com

${ }^{2}$ Division of Pediatric, Department of Woman and Child Health and Public Health, Fondazione Policlinico Universitario A. Gemelli, IRCCS, Rome, Italy Full list of author information is available at the end of the article
}

Autosomal recessive inheritance are associated with mutations in the COL9A1 gene and LOXL3 gene. Penetrance is complete, but phenotypic variability occurring both within and among families.

The full list of genes responsible for SS and associated phenotypes include: COL2A1, COL11A1, COL11A2, COL9A1, COL9A2, COL9A3, LOXL3 and PLOD3.

This variability makes the diagnostic approach difficult and for this reason the incidence of the syndrome can be significantly underestimated, approximately one in 7500-9000 neonates [2].

The typical clinical manifestations of SS are: I) ocular involvement with findings of congenital or early onset 
cataract, congenital abnormalities of the vitreous humour, retinal detachment and myopia greater than 3 diopters; II) sensorineural or conductive hearing loss; III) midfacial underdevelopment, and cleft palate (as part of the Pierre Robin sequence); IV) joint involvement with mild spondyloepiphyseal dysplasia, joint hypermobility and early onset arthritis [2].

Patients with the syndrome - especially COL11A2 gene mutation - may exhibit short stature.

Association between Stickler's syndrome and Basedow-Graves' disease or other thyroid dysfunction has never been reported in literature.

Basedow-Graves's disease, hypothyroidism and autoimmune thyroiditis with early onset were already described in other genetic syndromes such as Turner syndrome (TS) [3] and Down syndrome (DS) [4, 5].

Basedow-Graves' disease is uncommon in the pediatric age (especially in children younger than 6 years old) and associated with developing complications due to a possible late diagnosis [6].

The initial clinical presentation is often severe, especially if the child is young and/or pre-pubescent.

We report the case of a 5-years old child affected by type I Stickler syndrome, in which the diagnosis of Graves's disease was performed in preclinical state during health supervision and general nutritional assessment [7].

\section{Case presentation}

This is a girl affected by Stckler's syndrome, with clinical and molecular confirmation (mutation c872G > T, p.Gly291Val of COL2A1 gene) in follow up at the Rare Diseases Unit of Fondazione Policlinico Universitario A. Gemelli - IRCCS. The syndrome has maternal segregation.

At 5 years her weight, height, and head circumference were at 10th, 5th percentile and 50th percentile, respectively. Neurological and behavioral assessment did not reveal abnormalities. Eye examination revealed moderate myopia (8/10 diopters). Her hearing responses were intact. The tympanogram showed a slight depression and mild asymmetry of the cocleo-stapedial reflexes. She presented a mild generalized joint hypermobility.

Her vital signs were normal without signs of seizures, tremor, or psychomotor abnormalities. No hepatomegaly, splenomegaly were found.

For short stature, bone age was detected and resulted equivalent to her chronologic age. Laboratory studies revealed a normal levels of hemoglobin concentration, leukocyte and platelet count, aspartate aminotransferase and alanine aminotransferase levels.

Her thyroid-stimulating hormone (TSH) level was $<0.01$ microIU/mL (normal range, 0.5-4.8 $\mathrm{IU} / \mathrm{mL}$ ), free thyroxine (free T4) was $30.3 \mathrm{pg} / \mathrm{ml}$ (vn 8.5-16. 5). Thyroid ultrasound showed for a picture of thyroid glandular hyperfunctions (increase of glandular size, inhomogeneous structure and increase of blood flow).

The patient was referred to the Endocrine Pediatric Unit of our Polyclinic for the appropriate appraisals and treatments. The tests confirmed the diagnosis of BasedowGraves' disease [TSH $<0.01$ microUI / ml; triiodothyronine (T3) $19.9 \mathrm{pg} / \mathrm{mL}$; FT4 $42.1 \mathrm{pg} / \mathrm{mL}$; Anti-thyroperoxidase antibodies: > 1300 IU / mL (vn < 60); Anti-receptor antibodies of TSH (TRAb): 13.97 IU/L (vn < 1.75)].

Screening for other autoimmune diseases was performed (total IgA and antitransglutaminase antibodies to exclude celiac disease; GAD autoantibodies (GADA) to exclude type I diabetes mellitus) and it was negative.

As indicated by the paediatric endocrinology consultant, she was treated with Methimazole (MMI) with an initial dose of a half a $5 \mathrm{mg}$ tablet three times a day $(0.45 \mathrm{mg} / \mathrm{kg} /$ day $)$. Other drugs, such as $\beta$-blockers, were not added in absence of cardiological signs or symptoms, according to the literature [8].

After 5 days of therapy with good tolerance, the child was discharged and a short-term endocrinological follow-up was scheduled. Dose scaling is currently on going in reason of the progressive normalization of thyroid function.

\section{Discussion and conclusions}

Graves's disease (GD) is the commonest cause of hyperthyroidism, $84 \%$ of paediatrics cases. It is a rare disease, especially in childhood, accounting for 1 to $5 \%$ of all patients with GD. It may occur at any age during childhood, but its frequency increases with age, peaking during adolescence. Its incidence is estimated around 0.1 cases per 100.000 individuals in prepubertal children and 3 cases per 100.000 during adolescence [4].

$\mathrm{GD}$ is a form of autoimmune hyperthyroidism where the thyroid is stimulated to produce excess hormones by TSH receptor antibodies (TRAb) [9]. Other more specific signs are thyromegaly, and exophthalmos, that is detectable in only $20 \%$ of cases, whilst it is generally considered as a typical clinical sign of GD in adulthood $[4,9]$. Ocular signs are generally less severe in children than in adults and can be attributed to the inflammation and muscle swelling rather than to infiltrative disease of the orbital structures $[4,10]$. As expected, these signs (lid retraction and eye proptosis) may spontaneously improve in the majority of children after euthyroidism restoration $[4,10]$. In addition, pretibial myxedema is more infrequent in children than in adults $[4,10]$. Typical of pediatric GD is the acceleration of linear growth and bone maturation associated with prolonged hyperthyroidism $[4,11]$. These clinical manifestations are due to stimulating antibodies directed against antigens common to the thyroid and extra-thyroid tissues (ocular and extraocular muscles, adipocytes, fibroblasts) [4]. 
Our patient received the diagnosis of GD in preclinical state, during health supervision and evaluation by pediatric endocrinologist for short stature. This allowed us to make early diagnosis of GD thus starting therapy as early as possible with the benefits that derive from it. Furthermore, the diagnosis of GD gave us the possibility of a personalized management of our patient with SS. In fact, one of the manifestations of the SS is the ocular involvement that also characterizes the clinical picture of the GD. Having learned that our little patient is suffering from GD, we have personalized the endocrinological and ophthalmological follow-up taking into account the possible ocular changes that the girl can develop not only because of the SS but also because of the GD.

GD diagnosis was based on detecting a suppression of serum TSH concentrations and the presence of antiTSH receptor antibodies, according to the literature [8].

Although Graves' disease is by far the most common cause of children' hyperthyroidism, nevertheless other pathologies may be less frequently involved in its etiology. Hashimoto's thyroiditis, McCune Albright syndrome, toxic thyroid adenoma, thyroid cancer, subacute thyroiditis, TSH-secreting pituitary adenomas, pituitary thyroid hormone resistance, high levels of HCG and the ingestion of exogenous thyroid hormone are other causes of thyrotoxicosis in childhood. Except for Hashimoto's thyroiditis which can often be confused with Graves-Basedow's disease in thyrotoxicosis phase, the other causes of children' hyperthyroidism do not usually present differential diagnosis problems [6]. The distinguishing mark of Graves' disease is the presence of antibodies to the TSH receptor (TSHR antibodies; TRAb) while autoimmune thyroiditis will typically have thyroid peroxidase antibodies present but not TRAb. Antithyroglobulin and anti-thyroperoxidase antibodies are often found to be increased (in $60-80 \%$ of cases of Graves's disease), as a result of thyroid tissue damage [12].

Graves's disease is predominant in girls, in children with other autoimmune diseases (linked mainly to Type 1 Diabetes, Turner's syndrome, Down Syndrome, Di George Syndrome) and in children with a family history of autoimmune thyroid disease (AITD). Inherited forms account for 15 to $20 \%$ of cases (1st degree) [3-5, 13, 14].

A significant association between GD related hyperthyroidism and syndromic patients has already been described in literature for Turner syndrome (TS) [3] and Down syndrome (DS) $[4,5]$. The prevalence of both HT and GD was reported to be significantly higher in TS girls than in the paediatric general population: respectively $10-20 \%$ vs about $1.2 \%$ for HT and $1.7-3 \%$ vs $1.07 \%$ for GD [15]. In DS children, HT is the most common autoimmune disease and its prevalence has been reported to be much more elevated than in age-matched patients without this condition: respectively $13-34 \%$ vs
1.3\%. In DS children, also the prevalence of GD is known to be higher than in the paediatric general population: respectively $6.5 \%$ vs $1.07 \%$ [16].

Our patient is female with negative family history of autoimmune or thyroid disease. In addition, she has a personal negative medical history of autoimmune disease. We suspect that SS may have a role as risk factor in developing endocrine disease in pediatric age for two main factors: - the rarity of genetic condition (SS); the rarity of endocrine disease in pediatric age (GD) in the absence of suggestive family history.

With our work we want to report - for the first time the possibility of occurrence of Graves' disease among the clinical manifestations of Stickler syndrome type 1.

Further diagnoses of Graves' disease and or other immunity and endocrinological abnormalities are expected before suggesting the implementation of new clinical skills for Stickler syndrome.

In the meantime, in case of suspect, this paper may contribute to improve personalized management of this rare disease.

\section{Abbrevations \\ SS: Stickler syndrome; TS: Turner syndrome; DS: Down syndrome; \\ TSH: Thyroid-stimulating hormone; MMI: Methimazole; GD: Graves's disease; TRAb: Antibodies to the TSH receptor; AITD: Autoimmune thyroid disease; $H T$ : Hashimoto's thyroiditis}

\section{Acknowledgements}

Not applicable.

\section{Authors' contributions}

All authors have participated in the diagnostic pathways, all authors have read and approved he final manuscript.

\section{Funding \\ Not applicable.}

\section{Availability of data and materials}

Not applicable.

Ethics approval and consent to participate Not applicable.

\section{Consent for publication}

For the publication, the authors have obtained consent from the parents of both children.

\section{Competing interests}

The authors declare that they have no competing interests.

\section{Author details}

${ }^{1}$ Center for Rare Diseases and Birth Defects, Department of Woman and Child Health and Public Health, Fondazione Policlinico Universitario A. Gemelli, IRCCS, Rome, Italy. ${ }^{2}$ Division of Pediatric, Department of Woman and Child Health and Public Health, Fondazione Policlinico Universitario A. Gemelli, IRCCS, Rome, Italy. ${ }^{3}$ Division of Endocrinology and Metabolic Diseases, Catholic University of the Sacred Heart, Rome, Italy. ${ }^{4}$ Catholic University of the Sacred Heart, Rome, Italy. ${ }^{5}$ Division of Ophthalmology, Fondazione Policlinicico Universitario A. Gemelli, IRCSS, Roma, Italy. ${ }^{6}$ Pediatric Neurology Unit, Catholic University of Sacred Heart, Fondazione Policlinico Agostino Gemelli IRCCS, Rome, Italy. ${ }^{7}$ National Centre of Services and Research for the Prevention of Blindness and Rehabilitation of Low Vision Patients - IAPB Italia Onlus, Rome, Italy. 
Received: 22 April 2020 Accepted: 24 November 2020

Published online: 01 December 2020

\section{References}

1. Nathaniel HR, Rocio TM, Leena AK. Stickler syndrome. Gene reviews; 2017.

2. Higuchi Y, Hasegawa K, Yamashita M, Tanaka H, Tsukahara H. A novel mutation in the COL2A1 gene in a patient with stickler syndrome type 1: a case report and review of the literature. J Med Case Rep. 2017;11:237.

3. Valenzise M, Aversa T, Corrias A, Mazzanti L, Cappa M, Ubertini G, et al. Epidemiology, presentation and long-term evolution of Graves' disease in children, adolescents and young adults with Turner syndrome. Horm Res Paediatr. 2014;81:245-50

4. Zirilli G, Velletri MR, Porcaro F, Di Giovine G, Maisano P, La Monica G. Hyperthyroidism in childhood: peculiarities of the different clinical pictures. Acta Biomed. 2015:86(3):220-5

5. Goday-Arno A, Cerda-Esteva M, Flores-Le-Roux JA, Chillaron-Jordan JJ, Corretger JM, Cano-Pérez JF. Hyperthyroidism in a population with Down syndrome (DS). Clin Endocrinol. 2009;71:110-4.

6. Espinosa-Muñoz E, Ramírez-Ocaña D, Martìn-Garcìa AM, Ruiz-Garcìa FJ. Clinical note Graves' disease in a 3 year-old patient with agranulocytosis due to anti-thyroid drugs: radioiodine ablation therapy as an effective alternative. Rev Esp Med Nucl Imagen Mol. 2017;36:260-2.

7. Romano C, van Wynckel M, Hulst J, Broekaert I, Bronsky J, Dall'Oglio L, et al. European Society for Paediatric Gastroenterology, Hepatology and Nutrition guidelines for the evaluation and treatment of gastrointestinal and nutritional complications in children with neurological impairment. J Pediatr Gastroenterol Nutr. 2017;65:242-64.

8. Harvengt J, Boizeau P, Chevenne D, Zenaty D, Paulsen A, Simon D, et al. Triiodothyronine-predominant Graves' disease in childhood: detection and therapeutic implications. Eur J Endocrinol. 2015;172:715-23.

9. Jonak O, Polubok J, Barg E. Graves' disease in 2.5 years old girl-6-years-long observation. Pediatr Endocrinol Diabetes Metab. 2016;22(2):76-9.

10. Grüters A. Ocular manifestations in children and adolescents with thyrotoxicosis. Exp Clin Endocrinol Diabetes. 1999;107(Suppl 5):S172-4.

11. Wong GW, Lai J, Cheng PS. Growth in childhood thyrotoxicosis. Eur J Pediatr. 1999;158:776-9.

12. Van Veenendaal NR, Rivkees SA. Treatment of pediatric graves' disease is associated with excessive weight gain. J Clin Endocrinol Metab. 2011;96:3257-63.

13. Michels AW, Eisenbarth GS. Immunologic endocrine disorders. J Allergy Clin Immunol. 2010;125:S226-37.

14. Lombardo F, Messina MF, Salzano G, Rabbone I, Lo Presti D, Calcaterra V, et al. Prevalence, presentation and clinical evolution of Graves' disease in children and adolescents with type 1 diabetes mellitus. Horm Res Paediatr. 2011;76:221-5

15. Aversa T, Gallizzi R, Salzano G, Zirilli G, De Luca F, Valenzise M. Atypical phenotypic aspects of autoimmune thyroid disorders in young patients with Turner syndrome. Ital J Pediatr. 2018;44:12.

16. Aversa T, Crisafulli G, Zirilli G, De Luca F, Gallizzi R, Valenzise M. Epidemiological and clinical aspects of autoimmune thyroid diseases in children with Down's syndrome. Ital J Pediatr. 2018:44:39.

\section{Publisher's Note}

Springer Nature remains neutral with regard to jurisdictional claims in published maps and institutional affiliations.

Ready to submit your research? Choose BMC and benefit from:
- fast, convenient online submission
- thorough peer review by experienced researchers in your field
- rapid publication on acceptance
- support for research data, including large and complex data types
- gold Open Access which fosters wider collaboration and increased citations
- maximum visibility for your research: over 100M website views per year
At BMC, research is always in progress.
Learn more biomedcentral.com/submissions

